\title{
OFFEN GESAGT. BESCHWERDEN EINES ARCHIVARS
}

\author{
von Herbert Hrachovec
}

Zusammenfassung: Open Access Archive für Fachdisziplinen unterscheiden sich von organisationseigenen Repositorien durch ihre geringere institutionelle Verankerung und konstitutive Translokalität. Aus ihrer Sicht fallen manche Schwierigkeiten der Open Access-Initiative besonders ins Gewicht. Am Beispiel eines kleinen disziplinären Repositoriums Sammelpunkt (für Philosophie) werden zwei Punkte hervorgehoben. Erstens finden sich Journalbeiträge (bisweilen in unterschiedlichen Versionen) immer häufiger in mehreren, offenen oder gebührenpflichtigen, Archiven. Und zweitens können spezialisierte Sammlungen der Anziehungskraft dominanter Portale im akademischen sozialen Netz nichts entgegensetzen. Die Gründe dieser Entwicklung sind zu analysieren. Eine konzeptuelle Unzulänglichkeit liegt darin, dass die publikumswirksame Rede vom offenen Zugang zur Forschungsliteratur bei näherem Hinsehen zu undifferenziert ist, um unbeabsichtigte Praktiken auszuschließen.

Schlüsselwörter: disziplinäre Open Access Archive; unkontrollierte Verbreitung wissenschaftlicher Literatur; para-legaler akademischer Dokumentenhandel

\section{SPEAKING OPENLY. COMPLAINTS OF AN ARCHIVIST}

Abstract: Disciplinary Open Access repositories differ from institutional ones in at least two important respects: the contributions they cover are restricted to an academic field, but necessarily trans-local in scope. Some difficulties that have recently been impeding the Open Access Initiative carry particular weight as far as these repositories are concerned. Two issues in particular will be discussed by referring to examples taken from a small philosophical repository Sammelpunkt. Firstly academic papers can (quite often in different versions and under various terms of use) increasingly be found in open as well as closed archives. Secondly, disciplinary repositories are overshadowed by the ubiquity of academic social web portals. One factor supporting these developments is the conceptual inadequacy of the catchphrase "Open Access", which cannot prevent applications unintended by the initial inventors of this phrase.

Keywords: disciplinary Open Access archives; uncontrolled paper dissemination; para-legal academic file sharing 
DOI: http://doi.org/10.31263/voebm.v71i1.1977

\section{Inhalt}

1. Einleitung

2. Heile Welt und dunkle Wolken

3. Verteilungskrampf

4. Sozialstationen

5. Zweifelhafte Aussichten

6. Gegendarstellung

\section{Einleitung}

Die überwiegende Mehrzahl der im Directory of Open Access Repositories (http://www.opendoar.org) erfassten österreichischen Open Access Archive wird von Universitäten oder universitätsnahen Institutionen angeboten. Etwa ein Drittel betreuen Initiativgruppen, Arbeitskreise und Kompetenzzentren. Institutionelle und disziplinär orientierte Archive bedienen sich derselben Technik, nämlich des von der Open Archives Initiative entwickelten Protokolls für Metadata Harvesting $(\mathrm{OAI}-\mathrm{PMH})^{1}$ ( siehe nächster Abschnitt).

Sie unterscheiden sich jedoch in der Organisationsform und der anvisierten Klientele. Hochschulleitungen sind bestrebt, die wissenschaftlichen Aktivitäten ihrer Angestellten zu dokumentieren und bereitzustellen, während themenzenterierte Repositorien definitionsgemäß institutionsübergreifend organisiert sind. Das Service und die Öffentlichkeitswirksamkeit der beiden Typen differieren entsprechend. Etablierte Forschungseinrichtungen verfügen in der Regel über ein Basisbudget und erfüllen einen öffentlichbildungspolitischen Auftrag, während die Akteure, die sich für das Archiv einer Einzeldisziplin engagieren, die Organisationsstruktur für ihre gemeinsamen Interessen erst finden müssen. (Übersichten und Spezifika disziplinärer Archive diskutieren u.a.: [Xia, 2007], [Adamick and Reznik-Zellen, 2010], [Puplett, 2010], [Erway and OCLC Research, 2012], [Vierkant, 2013], [Tay, 2016]).

Ein kleines Fachrepositorium, wie der vom Autor eingerichtete Sammelpunkt (http://sammelpunkt.philo.at), trägt keine den institutionellen Archiven vergleichbare Verantwortung, ist aber andererseits stärker von der Informationsökologie des Internets abhängig. Während eine Universität 
jedenfalls auf die gesammelte Produktivität ihrer Mitglieder zurückgreifen kann, ist der Beitrag eines disziplinären Repositoriums, abgesehen von einigen prominenten Einzelfällen, weniger prominent. Er ist auch stärker mit der konfusen Dissemination wissenschaftlicher Arbeiten konfrontiert, die in jüngster Zeit im globalen Informationsaustausch zu beobachten ist. Anmerkungen zu dieser Entwicklung, ausgehend von Beispielen aus Sammelpunkt, sind allerdings auch für komprehensiver angelegte Portale von Interesse. Die Diskussion um deren Aufgabenbereich dreht sich oft um die (mangelnde) Bereitschaft der Wissenschaftlerinnen zur Open Access-Archivierung und um verschiedene Anreize (sowie Zwangsmaßnahmen), dieser Trägheit abzuhelfen ([Neylon, 2008], [Salo, 2008], [Covey, 2011], [Poynder, 2017]). Aus Sicht einer Ein-Mann-Initiative werden einige Stellen deutlicher, an denen die erfolgreich entwickelten Organisationsstrukturen des Open Access von neuen Entwicklungen im Bereich sozialer Netzwerke in Frage gestellt werden.

\section{Heile Welt und dunkle Wolken}

Die technische Infrastruktur zum automatisierten Informationsaustausch zwischen Open Access-Archiven, auf den die sozio-politischen Initiativen des freien Zugangs zur Forschungsliteratur aufbaut, wurde ab 1999 entwickelt, um den Datenbestand einzelner disziplinärer Repositorien kompatibel zu machen. Das in der Folge entwickelte „Open Access Initiative - Protocol for Metadata Harvesting“ (OAI-PMH) ermöglicht die bibliographische Auswertung standard-konformer Archive und die Aggregation ihrer Inhalte in umfassendere digitale Bibliotheksbestände. Der Impuls der Budapester Erklärung von 2002 (sowie der daran anschließenden Deklarationen) http:// www.budapestopenaccessinitiative.org/) bestand darin, die technische Machbarkeit einer neuen „alexandrinischen Bibliothek“ mit einem Appell zur Befreiung des wissenschaftlichen Publikationswesens aus der Abhängigkeit kommerzieller Verlagshäuser zu verbinden. Lokale Initiativen dienen dieser Vereinbarung nach als Sammelstellen, die ihre Bestände über eine spezielle Schnittstelle für „Harvester“ zur Verfügung stellen. „Service Providers“ bereiten dieses Material weiter auf und bereichern es mit Serviceangeboten. Der traditionelle freie Austausch von Belegexemplaren unter Wissenschaftlerinnen, im Verbund mit passenden Übertragungsprotokollen, sollte auf diesem Weg eine neue Ära akademischer Selbstbestimmung einleiten.

Tatsächlich wurde in zahlreichen Forschungsfeldern ein zweistufiges System aufgebaut, das Einreichungen in Einzelarchiven in leistungsfähige Portale kanalisiert. BASE, OpenAIRE und CORE sind Beispiele für solche 
professionell gewarteten Kollektionen, in denen die Suche nach frei zugänglicher Fachliteratur und die Entdeckung inhaltlich benachbarter Arbeiten in globalem Maßstab benutzerfreundlich verläuft ${ }^{2}$. Bo-Christer Björk hat 2013 einen Forschungsbericht über „Open Access Subject Repositories: An Overview" vorgelegt, in dem er überblicksartig 86 Archive für spezielle Disziplinen vorstellt [Björk, 2014]. Sieben von ihnen enthalten über 100.000 Datensätze, die Mehrzahl (21 Initiativen) fällt in die Größenordnung zwischen 1.000 bis 10.000 Einträgen. Nur die umfangreichsten Portale (darunter PubMedCentral, arXiv und SSRN ${ }^{3}$ ) können den Anspruch erheben, einen repräsentativen Teil ihrer Fachwelt zu erfassen. Der Rest ist durch das skizzierte MetadataHarvesting in die Distribution der genannten Service Provider eingebunden. Soweit, in der Planskizze, die heile Welt einer Wissenschaftslandschaft, deren Angehörige ihre Publikationen mit Hilfe automatisierter Prozeduren den Kolleginnen und der Öffentlichkeit zur Verfügung stellen.

Bekanntlich haben sich die Erwartungen an eine Emanzipation der Wissenschaftsliteratur vom kommerziellen Unterbau des Verlagswesens nur stellenweise erfüllt. Diese Entwicklung, die beide Typen von OA-Repositorien betrifft, ist hier nicht das Thema. Stattdessen sollen zwei Faktoren hervorgehoben werden, die sich für ein kleines, fachspezifisches Archiv wie Sammelpunkt besonders störend auswirken. Der eine Punkt betrifft die einzelnen Einreichungen. Es stellt sich nämlich heraus, dass die Distribution im digitalen Netzwerk in gewisser Hinsicht zu gut funktioniert. Im methodisch abgegrenzten Rahmen des OAI-PMH-Protokolls ist die Verbreitung effektiv geregelt. Eine Archivquelle verwaltet das eingereichte Dokument, während die übergreifenden Instanzen dessen Metadaten vorhalten und für den Inhalt auf die Herkunftsstelle zurückverweisen. Die Wirklichkeit sieht anders aus. Es besteht keine Kontrollinstanz, die verhindern könnte, dass eine digitale Einreichung nicht in verschiedenen Versionen und über unterschiedliche Distributionsnetze verteilt wird. Intuitiv ist es verständlich: Warum soll jemand nicht, wenn es schon um Open Access geht, sein Produkt an möglichst viele Stellen platzieren? Für institutionelle Archive ist das Problem weniger dringlich, weil die betreffenden Einrichtungen primär auf die Dokumentation ihrer Angehörigen ausgerichtet sind. Für Fachrepositorien erweist sich die Tendenz als bedenklicher.

\section{Verteilungskrampf}

Ein Beispiel verdeutlicht die Situation. Die Universität Craiova (Rumänien) publiziert eine philosophische Zeitschrift „Analele Universităţii bin Craio- 


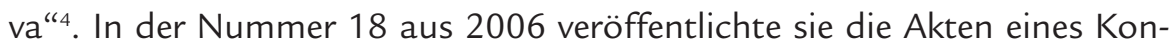
gresses über Martin Heidegger und die Philosophie des 20. Jahrhunderts. Die gescannte Fassung der gesamten Publikation wurde im Rahmen einer Forschungszusammenarbeit mit dem Institut für Philosophie an der Universität Wien mit Zustimmung der Organisatorinnen in Einzelbeiträge zerlegt, für jeden Artikel mit Metadaten versehen, und in das disziplinäre Archiv Sammelpunkt aufgenommen (2013). Den einleitenden Beitrag der Akten verfasste Alexandru Boboc: „heideggers ontologie und die neuren anwendungen der phänomenologie und der hermeneutik“ (sic!) ${ }^{5}$. Unbemerkt blieb (vermutlich), dass Alexandru Boboc genau denselben Beitrag ebenfalls 2006 in einer zweiten rumänischen Zeitschrift, nämlich „Cultura. International Journal of Philosophy of Culture and Axiology" veröffentlicht hatte (https://is.gd/DN7inf). Die Version in Analele weist im Vergleich dazu zwei geringfügige Unterschiede auf. Ihr ist ein Abstrakt in französischer Sprache beigefügt und ihr Titel, in idiosynkratischer Kleinschreibung gehalten, enthält ein fehlerhaftes „neure“. In Cultura ist zu lesen: „Heideggers Ontologie und die Neuen Anwendungen der Phänomenologie und der Hermeneutik"6. Die Differenz ist von sozusagen biblio-genetischem Interesse, weil die beiden Publikationen am Beginn separater Verteilungsverläufe stehen.

Die Zeitschrift Cultura wird online vom „Philosophy Documentation Center" angeboten. Das Copyright ihrer Beiträge liegt beim Journal, das eine Veröffentlichung (ausgenommen die Druckfassung) auf „open access websites“ gestattet. Für das Cultura-Original zahlt man USD 20.-. Auf diese Ressource verweist der einschlägige bibliographische Eintrag in PhilPapers (https://philpapers.org/rec/BOBHOU), der auf die erste (Probe-)Seite im „Documentation Center" verlinkt. Auch in Researchgate (https://is.gd/kPCMIF) findet sich die Literaturangabe, in diesem Fall verbunden mit einem Schaltknopf („Request full text“), der zu einer Abfrage der Benutzerdaten führt. Sie dient, nach der Erklärung des Portal, dazu, den Autor um eine Kopie des Textes zu bitten. Und schließlich kann man auf einer Webseite unbestimmter Urheberschaft ein „Open Access Journal“ mit dem kompletten Inhaltsverzeichnis der Cultura-Jahrgänge 2005-2010 aufrufen, verlinkt auf die dazugehörigen Texte. (http://oajournals.blogspot.co.at/2011/02/cultura-international-journal-of.html) Die Verweise führen jedoch ins Leere, genauer gesagt den 404 („,nicht gefunden“) Fehlerkode eines in Singapur registrierten „International Journal(s) of Axiology“. In Summe kostet der annoncierte Artikel also USD 20.- oder (nach dem Prinzip Hoffnung) eine Registrierung bei Researchgate. Es sei denn, man hält sich an die praktisch identische, von der Eigentümerin des Copyrights freigegebene, Fassung im Open Access-Repositorium. 
Auch diese Quelle hat eine Spur gezogen. Auf DOCPLAYER (https:// is.gd/7df8Vw) findet sich die in Sammelpunkt aufgenommene Analele-Fassung - leicht am französischen Abstrakt und am Schriftbild des Titels zu erkennen. Für einen Download muss die Leserin zunächst in einem Bildtest beweisen, dass sie kein Roboter ist, worauf sie das integrale SammelpunktDokument erhält. Zusätzlich bietet diese Ansicht auch eine gänzlich verfehlte Konvertierung des PDF-Dokumentes in ASCII-Text, in welcher die Fußnoten (und sogar die Seitenzahlen) unabgesetzt in den Textfluss einbezogen sind. Der Download und das verstümmelte „Transkript“ lassen sich (ein Extra-Service) über den Kode eines bereitgestellten iFrames in beliebige Webseiten einblenden.

Die vorgestellte Recherche erscheint vielleicht beckmesserisch, aber ein Archivar kann sich fragen: „Wer soll sich die Mühe antun, derartige Beiträge sachgerecht für Open Access aufzubereiten und bereitzustellen?" Ringsumher finden sich kommerzielle, para-legale, verschlampte und nur mehr nominell existierende Kanäle, durch welche das Material transportiert wird.7 Sicher, es ist eine gewisse Befriedigung "to do the right thing“. Aber als Fels in der Brandung vielfältiger Vertriebsoptionen ist Open Access ursprünglich nicht aufgefasst worden. Der akademische Wissenstransfer kann und muss, sollte man meinen, für sich selber sorgen. Dieses Prinzip treibt im Internet seltsame Blüten.

\section{Sozialstationen}

Das zweite angekündigte Problemfeld ist in der vorigen Darstellung bereits angedeutet. Researchgate und DOCPLAYER sind Social-Web-Entwicklungen, die Forscherinnen einladen, ihre Arbeiten in der Erwartung bekannt zu machen, dass sie Gleichgesinnte und Interessentinnen erreichen. Die Portalbetreiber sammeln die Transaktionsdaten und erstellen daraus Profile, Trends und Expertisen mit Marktwert. Der im Moment größte Anbieter solcher Dienstleistungen ist academia.edu, wo momentan - um beim oben genannten Beispiel zu bleiben - allerdings nur ein von Alexandru Boboc mitherausgegebener Sammelband zur Philosophie des 19. Jahrhunderts verfügbar ist. Allerdings zeichnet sich das Portal in anderer Hinsicht aus. Es informiert darüber, dass es 1.004 Dokumente enthält, in denen der Name „Alexandru Boboc" vorkommt ${ }^{8}$. Einsehbar ist die Information nach dem Upgrade auf einen premium account für EUR 89.- im Jahr. (Gratis sind mehrere Emails mit Boboc-bezogenen Mitteilungen in den nächsten Tagen.) Das Geschäftsmodell ist überaus erfolgreich. Wissenschaftlerinnen werden weltweit von den 
Netzwerkeffekten der bedienungsfreundlichen, ihren persönlichen Bedürfnissen angepassten, Plattform angezogen und zahlen für die nutzbringende Aufbereitung von Daten(verläufen), die sie selbst, mit ihren Aktivitäten innerhalb des Portals, erzeugen. Vor den wissenschafts-ethischen Bedenken, die sich rasch melden, ein Caveat. Die Benutzerdaten, um welche es letztendlich geht, werden von academia.edu bloß klug attrahiert und kanalisiert. Auch eine Statistiksoftware im Hintergrund der eigenen Webseite, die Logfiles des Universitätsservers, oder die Auswertung von Blogzugriffen erfassen den relevanten Datenverkehr. Die Kunst liegt darin, ihn der Profession, deren Verfahren sie strukturell abbilden, als Mehrwert anzubieten.

Die großen Institutionen der Forschungsförderung, die Open Access zu den von ihnen subventionierten Arbeiten verlangen, sowie die Wissenschaftsministerien und Universitätsleitungen, die entsprechende Mandate festlegen, demonstrieren damit ihre Tätigkeit im Interesse eines nicht von kommerziellen Interessen gesteuerten Wissenschaftsbetriebs. Die Daseinsberechtigung ihrer Repositorien ist zumindest teilweise von den beschriebenen Verbreitungsmodalitäten unabhängig. Wenn andererseits die Suche nach Alexandru Boboc' Artikel auf Google Scholar das PDF mit Volltext in seiner Sammelpunkt-Version aus dem CORE Aggregatarchiv verlinkt, wird deutlich, dass die Erschließung dieses speziellen Dokuments in der großen Suchmaschine unsichtbar wird. ${ }^{9}$ Um noch einen Fall zu nennen: Violetta Waibels Studie „Wechselbestimmungen. Zum Verhältnis von Hölderlin, Schiller und Fichte in Jena." ist 2006 in Sammelpunkt aufgenommen und anschließend von CORE erfasst worden. Dorthin verweist auch Google Scholar. Aber die Suchmaschine hält sich an kein Prinzip. Eine Arbeit Waibels über Hölderlins Rezeption von Fichtes „Grundlagen des Naturrechts“ die ebenfalls in CORE erfasst ist, wird durch Google Scholar einem Archiv namens philo.at zugeordnet, hinter dem sich Sammelpunkt verbirgt.

Angesichts dieser Datenlage kann man es Wissenschaftlerinnen nicht verdenken, dass ihnen spezielle disziplinäre Repositorien gleichgültig sind; ihren Betreibern muss man raten, sich auf derartige Lotteriespiele einzustellen. Das Problem hat tiefe Wurzeln. Es beginnt damit, dass die klassischen Aufrufe zu Open Access weder Verlage, noch die charakteristischen Subkulturen ins Auge gefasst haben, welche die Wissenschaftspraxis ausmachen. Es ist schon in der Projektbezeichnung angelegt: „offen“ steht gegen „geschlossen“. Diese Alternative ist viel zu plakativ, um die Vorgänge innerhalb einer akademischen Disziplin und insbesondere hinsichtlich ihrer Publikationspraktiken zu erfassen. Es handelt sich dabei um Ergebnisse vielfältiger Interaktionen, in denen sich Expertise, Prestige, Interaktionen, ein Diskussionsstil und Kontrollmechanismen herausbilden. Einige Facetten davon 
können in sozialen digitalen Netzwerken unterstützt werden. Diese antworten auf ein Bedürfnis, welches Archivbestände, seien sie noch so verfügbar, nicht erfüllen können. Fachdisziplinen sind im Vergleich zu Universitäten weder lokal, noch administrativ fixiert, entsprechend informeller ist ihr sozialer Druck und auch die Kohäsion, die sie ihren Mitgliedern bieten können.

Gegeben die zunehmende Prekarisierung am akademischen Arbeitsmarkt, welche die Bindung an einzelne Hochschulen schwächt, sind Wissenschaftlerinnen verstärkt genötigt, sich nicht nur fachintern zu qualifizieren, sondern ein möglich attraktives Dossier ihrer beruflichen Leistungen und Kontakte zu erstellen. An dieser Stelle trifft das Interesse der Hochschulangestellten auf das Angebot akademischer Selbstdarstellungsportale. Der gute Rat zur Gründung einer „Ich AG“ mit den dazugehörigen Markenzeichen passt zur Situation. Es reicht nicht mehr, auf ein respektables Curriculum Vitae und eine eindrucksvolle Publikationsliste zu verweisen. Zusehends gehört auch Selbstvermarktung zum Job und dazu bieten die akademischen Social-Web-Portale reichlich Gelegenheit.

Natürlich ist die grenzüberschreitende Kommunikation seit jeher ein Charakteristikum von Wissenschaft. An diesen Umstand appellieren auch alle Aufrufe zur Einrichtung einer Wissens-Allmende. Problematisch wird es allerdings, wie sich nachträglich herausstellt, wenn die beschriebenen Nutzungsanwendungen der digitalen Informations- und Kommunikationstechnologie in der Entwicklung der Wissensökonomie überhandnehmen... Im vorigen Abschnitt ist beschrieben worden, auf welch sonderbaren Wegen ein unter anderem im Open Access verfügbarer Zeitschriftenbeitrag im Internet verbreitet wird. Die Reaktion auf diese Eventualität kann sich nicht auf die Maxime beschränken, nur „geprüfte Quellen“ zu konsultieren. Erstens ist nicht immer klar, nach welchen Kriterien das zu beurteilen ist, zweitens existieren keine standardisierten Qualitätsmessungen für Peer Review, drittens lassen sich Suchergebnisse und Statistiken unschwer manipulieren und viertens erfüllt die para-legal verbreitete Kopie einer wissenschaftlichen Arbeit, für die ja nicht die Rezipientin verantwortlich ist, denselben Zweck wie das Original.

\section{Zweifelhafte Aussichten}

Was folgt aus diesen Beobachtungen? Auf der Einstiegsseite der in Russland eingerichteten Library Genesis findet sich eine Solidaritätsbotschaft mit dieser riesigen Sammlung ungehindert zugänglicher wissenschaftlicher Literatur: „We have the means and methods to make knowledge acces- 
sible to everyone, with no economic barrier to access and at a much lower cost to society. But closed access's monopoly over academic publishing, its spectacular profits and its central role in the allocation of academic prestige trump the public interest."

Die Homepage von academia.edu erklärt ihrerseits: „Academia is the easiest way to share papers with millions of people across the world for free." ${ }^{10}$ So war "Open Access" nicht gedacht, aber das Adjektiv alleine kann das Problem nicht einmal andeuten. Anvisiert war an eine Bewegung gegen die ungezügelte Kommerzialisierung des wissenschaftlichen Publikationswesens. Sie ist nun von zwei Seiten bedrängt. Von Offenheit im Sinn von "Sammle alles, was Du irgendwie kriegen kannst“ und vom Geschäft der Lockangebote zum Abschöpfen von Daten. Nüchtern betrachtet hat die „reine Lehre“ der Open Access „Evangelisten“ ihre anfängliche Überzeugungskraft verloren.

Eine perspektivische Täuschung lag in der begrifflichen Assoziation zwischen „Open Source“ und „Open Access“. Programmkode unterscheidet sich von Wissenschaftsliteratur in entscheidenden Punkten. Er stellt keine Behauptungen auf, ist wesentlich auf Maschinen angewiesen, kann auf unterschiedliche Weise denselben Effekt erzielen und existiert als soziokulturell-politisches Phänomen seit etwas über einem halben Jahrhundert. Das Bibliothekswesen ist im Vergleich dazu uralt und an mächtige Interessensgruppen gebunden. Wissenschaftliche Publikationen einfach nach dem Vorbild der Copyleft Bestimmungen der GNU Lizenz zu betrachten, lenkt von den Schwierigkeiten der Sache ab.

Die Wahrnehmung der dargestellten Entwicklungen eröffnet unbequeme Aussichten. Ein Fixpunkt ist, wenn man das so sagen kann, das Schillern des Begriffes Offenheit. Einer der populärsten Beiträge in Sammelpunkt ist Zebunnessa Laizus (et al.) Arbeit über „The Role of ICT in Women's Empowerment in Rural Bangladesh“. Kopien des PDF Dokuments finden sich gemäß einer Recherche bei Google Scholar in insgesamt 11 Archiveinträgen ${ }^{11}$. Um sicher zu sein, dass man „richtig“ zitiert, muss man sie miteinander vergleichen. Die nachhaltige Verbreitung sei den Autorinnen gegönnt, aber sie ist keine Inspiration bei der Betreuung eines kleinen disziplinären Repositoriums. Zeit für einen Realitätscheck.

\section{Gegendarstellung}

Der vorliegende Artikel berichtet von einer Enttäuschung. Ihr Verlauf erinnert an einschlägige Erfahrungen mit großen Versprechen. Die mobili- 
sierende Kraft ambitionierter Entwürfe kann unter Umständen verpuffen. Zurück bleibt dann als Fazit Erinnerung und Ernüchterung. Das kann hier nicht so stehen bleiben. Es würde im günstigen Fall auf einen abgeklärten Pragmatismus oder auch auf Resignation hinauslaufen. Als wären die betroffenen Hoffnungspotenziale (Aufklärung, Selbstverwirklichung, globale Gerechtigkeit, entkommerzialisierter Zugang zur Wissenschaftsliteratur) die Mühe nicht wert. Kann der Impuls weit ausholender Appelle eine Berechtigung behalten, auch wenn seine Unzulänglichkeit zu beklagen ist? Auf die bedeutenden Erfolge der Open Access-Initiative in Öffentlichkeitsarbeit und Organisation ist hingewiesen worden. Wie ist mit den Fehlentwicklungen umzugehen? Das lässt sich nicht mit derselben Unbekümmertheit betrachten, wie die anfänglichen Parolen. Es ging um Beschwerden im Zusammenhang mit einem Spezialarchiv für Philosophie. Zur Antwort auf die eben aufgeworfenen Fragen kann die Philosophie einen Beitrag leisten.

Die problematischen Entwicklungen in den 15 Jahren seit der Budapester Erklärung sind quasi aus historischer Distanz beleuchtet worden. In dieser Einstellung geht es um Absichten zur Gestaltung wichtiger Facetten des menschlichen Zusammenlebens im Verlauf der Zeit. Damit verbindet sich häufig das Interesse, herauszufinden, wer (etwas) gewonnen oder verloren hat. Das zieht sich auch durch den Bericht des Archivars. Aber darin steckt eine unreflektierte Vorannahme. Die Aufmerksamkeit wird darauf gerichtet, welche Trends sich durchgesetzt haben und welche sich nicht halten konnten. Dagegen ist natürlich nichts zu sagen, aber man kann die Zusammenhänge anders sehen. Auch im Engagement für eine gute Sache gibt es eine Art life-work-balance. Ihr Augenmerk liegt nur zum Teil auf der Anstrengung, sie betont zusätzlich die Lebensqualität. Erstrebenswert ist danach eine positive Rückkopplung zwischen der Sache und den Menschen, die „ihr dienen“. Die Ausrichtung an einem öffentlichen Gut bereichert die Individuen. Wenn die Überzeugungskraft seines Auftretens nachlässt, bleiben - so das Desiderat - nicht "leere Kilometer" zurück, denn die Anstrengung war in einen Lebenslauf integriert. Enttäuschung ja, aber man ist nicht um persönlichen Gewinn betrogen worden.

Das ehrgeizige Vorhaben lässt sich, soviel ist deutlich, nicht in der angestrebten Form verwirklichen. Das ändert nichts daran, dass es ein Wunschziel bleibt und hindert nicht daran, die Arbeit neu zu konzipieren. Den objektivierten Entwicklungslinien, die Historikerinnen nachzeichnen, fehlt notgedrungen das Moment der Erfüllung, die handelnde Personen im Engagement für ein Ziel finden können. Die Wirklichkeitsnähe dieser abstrakten Reflexionen beleuchtet abschließend ein kleines informatisches Detail. 
Sammelpunkt läuft auf der Grundlage der seit 2000 an der University of Southhampton entwickelten Eprints Software. Deren Operationen sind im Einzelnen für einen mäßig fortgeschrittenen Anfänger in Unix Systemadministration viel zu komplex. Er muss dennoch nicht mit jedem Problem zu Informatikexperten fragen gehen. Die Arbeit mit dem Produkt wird durch eine Mailingliste unterstützt, welche die höchsten Ansprüche an fachlichkollegialer Kooperation im Internet erfüllt. Die umgehende, kostenlose Hilfe, die allen Betreibern der Software rund um die Uhr zur Verfügung steht, realisiert in einem mikroskopisch kleinen Teilbereich den Traum von gesellschaftlichen Verhältnissen, in denen die Arbeitsteilung keine unüberwindlichen Gräben zwischen Berufsgruppen schafft. Ein neugieriger Philosoph kann unter diesen Voraussetzungen ein professionelles Internet-Portal zur Archivierung frei zugänglicher wissenschaftlicher Arbeiten betreiben. Der Ausdruck „fachspezifisches Repositorium für Philosophie“ erhält damit eine zusätzliche Bedeutung.

ao. Univ.-Prof. i.R. Dr. Herbert Hrachovec

Universität Wien, Institut für Philosophie E-Mail: herbert.hrachovec@univie.ac.at

\section{Literatur}

[Adamick and Reznik-Zellen, 2010] Adamick, J. and Reznik-Zellen, R. (2010). Representation and Recognition of Subject Repositories. DLib Magazine, 16(9/10). DOI: http://doi.org/10.1045/september2010adamick

[Björk, 2014] Björk, B.-C. (2014). Open access subject repositories: An overview. Journal of the Association for Information Science and Technology, 65(4):698-706. DOI: https://doi.org/10.1002/asi.23021

[Boboc et al., 2015] Boboc, A., Bieg, B., Felton, R., Dalley, S., and Kravtsov, Y. (2015). Invited Article: A novel calibration method for the JET realtime far infrared polarimeter and integration of polarimetry-based lineintegrated density measurements for machine protection of a fusion plant. Review of Scientific Instruments, 86(9):091301. DOI: https://doi. org/10.1063/1.4929443

[Covey, 2011] Covey, D. T. (2011). Recruiting Content for the Institutional Repository: The Barriers Exceed the Benefits. Journal of Digital Information, 12(3). URL: https://journals.tdl.org/jodi/index.php/jodi/article/ view/2068 
[Duffy and Pooley, 2017] Duffy, B. E. and Pooley, J. D. (2017). "Facebook for Academics": The Convergence of Self-Branding and Social Media Logic on Academia.edu. Social Media + Society, 3(1):205630511769652. DOI: https://doi.org/10.1177/2056305117696523

[Erway and OCLC Research, 2012] Erway, R. and OCLC Research (2012). Lasting impact: sustainability of disciplinary repositories. OCLC Research, Dublin, Ohio. OCLC: 781442686. URL: http://www.oclc.org/research/ publications/library/2012/2012-03.pdf

[Neylon, 2008] Neylon, C. (2008). The trouble with institutional repositories. URL: http://cameronneylon.net/blog/the-trouble-with-institutional-repositories/

[Poynder, 2017] Poynder, R. (2017). The State of Open Access: Some New Data. URL: https://poynder.blogspot.co.at/2017/08/the-stateof-open-access-some-new.html

[Puplett, 2010] Puplett, D. (2010). Subject Repositories: European Collaboration in the International Context. Ariadne, (62). URL: http:// www.ariadne.ac.uk/issue62/bl-subject-repos-rpt/

[Salo, 2008] Salo, D. (2008). Innkeeper at the Roach Motel. Library Trends, 57(2):98-123. DOI: https://doi.org/10.1353/lib.0.0031

[Tay, 2016] Tay, A. (2016). 5 thoughts on open access, institutional and subject repositories. URL: http://musingsaboutlibrarianship.blogspot. co.at/2016/10/5-thoughts-on-open-access-institutional.html

[Vierkant, 2013] Vierkant, P. (2013). Focussing on disciplinary open access repositories. URL: http://open-access-to-gender-research.de/wpcontent/uploads/2013/08/Vortrag-Paul-Vierkant.pdf

[Xia, 2007] Xia, J. (2007). Disciplinary repositories in the social sciences. Aslib Journal of Information Management, volume 59:6, p. 528-538. DOI: https://doi.org/10.1108/00012530710839605

* Letzter Zugriff auf alle im Beitrag angeführten URLs: 21.05.2018

1 https://www.openarchives.org/pmh/

2 https://www.base-search.net/, https://www.openaire.eu/, https://core. ac.uk/.

3 https://www.ncbi.nlm.nih.gov/pmc/, https://arxiv.org/archive/physics, https://www.ssrn.com/en/

4 http://cis01.central.ucv.ro/analele_universitatii/filosofie/

5 http://sammelpunkt.philo.at/2308/

6 Die im Deutschen unstatthafte Großschreibung „Neuen“ entspricht den Regeln englischer Überschriften. 
7 Persistente Identifikatoren („persistent identifiers“), wie sie von OARepositorien angeboten werden können, verhindern die im Beispiel beschriebenen „Irrfahrten“. Leider ist dazu eine zusätzliche, zumeist kostenpflichtige, Infrastruktur nötig. Vgl. https://www.dpconline.org/ handbook/technical-solutions-and-tools/persistent-identifiers

8 Vermutlich hat den Journalbeitrag „A novel calibration method for the JET real-time far infrared polarimeter and integration of polarimetrybased line-integrated density measurements for machine protection of a fusion plant" nicht der Philosoph mit Namen „Alexandru Boboc“verfasst. [Boboc et al., 2015]

9 CORE selbst weist die Herkunft des Dokuments und seiner Metadaten nach. Diese Information geht beim direkten Verweis Google Scholars auf das digitale Endprodukt verloren.

10 [Duffy and Pooley, 2017] stellen den Zusammenhang zwischen den beiden „Offenheiten“ her: “At the core of the site's growth strategy (and revenue plans) is its massive trove of articles and chapters?- most of which are copyrighted. In that respect, Academia.edu, and its rival ResearchGate, are peer-to-peer PDF-sharing repositories, akin to Napster circa 1994. Another way to say this is that Academia.edu is like Sci-Hub, but with venture backing (and a carefully written, liabilitydodging 'Copyright Policy')."

11 Sammelpunkt ist dort nicht angeführt. 